\title{
Labor de trabajador social frente al trabajo infantil de los niños, niñas y adolescentes en el cantón Portoviejo
}

DOI: https://doi.org/10.33262/ap.v3i4.106

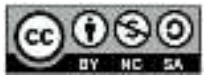

Work of social worker against child labor of children, girls and adolescents in canton Portoviejo

Mayra Isabel Mendoza Loor. ${ }^{1} \&$ Ligia Estela Loor Lino. ${ }^{2}$

\begin{abstract}
.
Introduction. Child labor is a latent problem in society, which brings with it a series of repercussions on the integral development of children and adolescents; in this sense, many efforts have been made to reduce and eradicate it. Objective. Characterize the work carried out by the Social Worker against Child Labor in children and adolescents of the Canton Portoviejo. The explanation of topics related to the study problem was carried out and was based on the review of the pertinent bibliography. Methodology. A descriptive research was applied, with a quantitative - qualitative approach, which led to the fulfillment of the proposed objectives. As techniques, the survey was applied by the inhabitants of the Parishes of Picoazá and San Pablo of the Cantón Portoviejo and the interview with the Social Worker of the Fundación Paso a Paso, the instrument was the question guide for the survey and the interview questionnaire. The population was 42 people and $100 \%$ of it was taken as a sample. Conclution. The action of the social worker in the face of this problem is important, since it articulates and prepares social reports, plans, leads coordination actions and intersectoral articulation for the restitution of the rights of children and adolescents in child labor, among others.
\end{abstract}

\footnotetext{
${ }^{1}$ Universidad Técnica de Manabí. Egresada de la escuela de Trabajo Social. mairymendoza@gmail.com, https://orcid.org/0000-0002-7850-1812

2 Universidad Técnica de Manabí. Docente auxiliar, tiempo completo, ligia.loor@utm.edu.ec, https://orcid.org/0000-0003-4651-2733
} 
Keywords: social work, child labor, children's rights, professional intervention.

\section{Resumen.}

Introducción. El trabajo infantil es una problemática latente en la sociedad, que trae consigo una serie de repercusiones en el desarrollo integral de los niños, niñas y adolescentes, en este sentido son muchos los esfuerzos que se han realizado por disminuirlo y erradicarlo. Objetivo. Caracterizar la labor que cumple el Trabajador Social frente al Trabajo Infantil en los niños, niñas y adolescentes del Cantón Portoviejo. Se realizó la explicación de temáticas referentes al problema de estudio y se fundamentó en la revisión de la bibliografía pertinente. Metodología. Se aplicó una investigación de tipo descriptiva, con un enfoque cuanti - cualitativo, lo que conllevó al cumplimiento de los objetivos propuestos. Como técnicas se aplicaron la encuesta los habitantes de las Parroquias de Picoazá y San Pablo del Cantón Portoviejo y la entrevista a la Trabajadora Social de la Fundación Paso a Paso, el instrumento fue la guía de preguntas para la encuesta y el cuestionario de la entrevista. La población fue de 42 personas y se tomó como muestra el 100\% de la misma. Conclusión. El accionar del trabajador social frente a esta problemática es importante, ya que articula y elabora informes sociales, planifica, lidera acciones de coordinación y articulación intersectorial para la restitución de derechos de los NNA en trabajo infantil, entre otros.

Palabras clave: trabajo social, trabajo infantil, derechos de los niños, intervención profesional.

\section{Introducción.}

El trabajo infantil es una problemática a nivel mundial, que se da por muchos factores entre ellos la pobreza en los hogares, los bajos salarios, el desempleo, enfermedades, la tolerancia social, entre otros. Históricamente las calles han sido uno de los escenarios donde se evidencian niños, niñas y adolescentes menores de 15 años trabajando hasta altas horas de la noche con el fin de contribuir con el gasto familiar, viéndose obligados la mayoría de ellos a dejar de estudiar y expuestos a riesgos que atentan su desarrollo integral.

En este sentido, las últimas estimaciones mundiales indican que 152 millones de niños (64 millones de niñas y 88 millones de niños) se encuentran en situación de trabajo infantil en todo el mundo, lo que representa casi uno de cada 10 de todos los niños del mundo (ILO, 2018).

De acuerdo a la Encuesta Nacional de Trabajo Infantil (ENTI) 2012 del Instituto Nacional de Estadística y Censos INEC:

De todos los niños/as y adolescentes de 5 a 17 años de edad, el 8,56\% realiza actividades de trabajo infantil. Del total de niños/as y adolescentes que trabajan, el $62,8 \%$ son hombres respecto al $37,2 \%$ que son mujeres. De todos los adolescentes de 15 a 17 años, el 15,7\% está en condición de trabajo infantil. Del 
total de niños/as y adolescentes en el área rural, el 15,5\% está en condición de trabajo infantil. El 60,4\% de los niños/as y adolescentes que trabajan lo hace por ayudar económicamente a su hogar, mientras que al $16,5 \%$ no le interesa la educación.

Por otro lado, “antes de la pandemia, Ecuador tenía 375.342 menores en situación de trabajo infantil. Eso significa al menos 10 estadios Olímpico Atahualpa llenos. Pero esa cifra se puede incrementar drásticamente con la pandemia por una razón: el desempleo de los adultos" (Morán, S 2020).

El trabajo que se ha venido realizando para disminuir, prevenir y erradicar esta problemática social ha sido arduo, el estado, los gobiernos locales, de turno y la sociedad en general han contribuido para que se diseñen planes, políticas y normativas que regulen el bienestar de este grupo de atención prioritaria.

Por su parte la constitución del Ecuador, en el art. 46, estipula que. - Protección especial contra cualquier tipo de explotación laboral o económica. Se prohíbe el trabajo de menores de quince años, y se implementarán políticas de erradicación progresiva del trabajo infantil. El trabajo de las adolescentes y los adolescentes será excepcional, y no podrá conculcar su derecho a la educación ni realizarse en situaciones nocivas o peligrosas para su salud o su desarrollo personal. Se respetará, reconocerá y respaldará su trabajo y las demás actividades siempre que no atenten a su formación y a su desarrollo integral.

En base a lo expuesto el objetivo de la investigación consistió en caracterizar la labor que cumple el Trabajador Social frente al Trabajo Infantil en los niños, niñas y adolescentes del Cantón Portoviejo, la siguiente información brinda conocimientos sobre las distintas formas de trabajo infantil y el accionar del profesional de Trabajo Social frente a esta problemática.

\section{Trabajo infantil}

De acuerdo a Jiménez K (2018) "El trabajo infantil es una problemática mundial, generada por la violación de los derechos de los niños, niñas y adolescentes, perjudicando su salud física, psicológica, sexual y deteriorando su estilo de vida”. (pág. 22). Del mismo modo la INEC (2015) lo define como aquella actividad que es peligrosa o dañina para los niños y niñas, interfiere con el aprendizaje formal o provoca deserción escolar prematura, requiere de combinar la escuela con una cantidad excesiva de trabajo, o no permite su desarrollo integral.

En concordancia con los autores anteriores, se entiende que el trabajo infantil es una problemática latente en la sociedad, en donde cotidianamente se puede ver a niños en los semáforos, en los mercados, en los parques, realizando alguna actividad a cambio de una mínima remuneración; actualmente, por la pandemia, se hizo más notorio el trabajo infantil puesto que el desempleo masivo de sus padres a causa de la emergencia sanitaria, ha despertado interés en contribuir con loa gastos del hogar. 
Por otra parte, Jaramillo, M (2017) menciona que el trabajo infantil es tan histórico como las civilizaciones, los niños aprendían de sus progenitores a desarrollar diferentes tipos de actividades, lo que permitía percibir que estaban preparaos para ejercer un trabajo. Así mismo Macri y Uhart (2017) agregan que algunos estudios socio-antropológicos dan cuenta de que el trabajo infantil se inscribe en las pautas naturales de crianza y socialización de las familias rurales, y la existencia de niños trabajadores se asocia a factores y características socioculturales de algunas poblaciones

En efecto, Pérez (2017) expresa que los niños han trabajado en cualquier clase de empleo: "los NNA han colaborado siempre en los procesos de producción y reproducción de la sociedad. Asimismo, han estado presentes en la mayoría de los sectores productivos, desde la manufactura, el comercio, los servicios y por supuesto en las actividades agrícolas".

Por consiguiente, Sandoval, A (2007) refiere sobre los efectos que tiene el trabajo infantil:

Los efectos económicos y sociales del trabajo infantil también son diversos e inciden tanto en el nivel microfamiliar como en el macroeconómico y social; a nivel microfamiliar, el trabajo infantil incrementa en el corto plazo el ingreso del hogar, pero en el largo plazo disminuye la formación de capital humano, ya que muchos niños y niñas que trabajan no asisten a la escuela, otros la abandonan antes de concluir la educación básica y unos más no continuarán estudiando más allá de este nivel.

Cabe mencionar que el constitucionalmente está prohibido el trabajo infantil, no obstante, se evidencia niños trabajando en el mundo entero, expuestos a diversos riesgos y desarrollando actividades que los adultos rechazaron por no contar con las condiciones laborales apropiadas. Nova (2008).

En base a estas consideraciones, se hace importante mencionar al código de la niñez y adolescencia, el art. 81 tipifica.- Derecho a la protección contra la explotación laboral.Los niños, niñas y adolescentes tienen derecho a que el Estado, la sociedad y la familia les protejan contra la explotación laboral y económica y cualquier forma de esclavitud, servidumbre, trabajo forzoso o nocivo para su salud, su desarrollo físico, mental, espiritual, moral o social, o que pueda entorpecer el ejercicio de su derecho a la educación.

Del mismo modo, en el art. 83.- manifiesta que, en la erradicación del trabajo infantil, el Estado y la sociedad deben elaborar y ejecutar políticas, planes, programas y medidas de protección tendientes a erradicar el trabajo de los niños, niñas y de los adolescentes que no han cumplido quince años. La familia debe contribuir al logro de este objetivo.

Llegados a este punto, Leyva, M., \& Pichardo, S. (2016) mencionan algo importante sobre esta problemática:

El hecho social de que los niños que trabajan cuenten con derechos que regulen las actividades que realizan es un peldaño más de colocación de límites para 
regular un tipo de trabajo que muchas veces, al ser desdeñado socialmente, sufre ya de una condición de fragilidad que afecta su dignidad humana y condición social; en este sentido son bienvenidos los derechos de los niños y, sin embargo, es un reconocimiento insuficiente que a veces puede llegar a tener efectos contraproducentes en culturas específicas.

\section{Accionar del trabajador social frente al trabajo infantil}

El trabajo infantil se constituye en uno de los múltiples pero privilegiado escenario de intervención trabajador social, en donde su rol está orientado a la protección social y restitución de derechos de los niños, niñas y adolescentes, intervención que amerita el uso adecuado un proceso metodológico de intervención que garantice el logro de los resultados esperados a favor del bienestar social de este grupo de atención prioritaria.

Al respeto, Cervantes (2017) realza la experiencia del Trabajo Social:

Sólo el área de Trabajo Social, por su experiencia en el trabajo de campo, palpa la realidad y tiene el conocimiento integral del contexto y de los entornos tan necesarios para desarrollar a profundidad el trabajo integral y responder eficientemente a la multifactorialidad presente en los casos de trabajo infantil. El Trabajo Social hace su presencia en el preciso momento donde lo privado se relaciona con lo público, con lo institucional, en donde lo personal y familiar es parte del conjunto.

Es así como el Trabajador Social se convierte en un agente mediador que prioriza los casos de vulnerabilidad infantil, desarrollando las funciones de funciones brindar atención directa, coordinar, prevenir y ejecutar estrategias comunicativas. Aragundi \& Menéndez (2020)

En este orden de ideas (Ochoa, 2020) menciona que situación de las y los trabajadores sociales es ardua porque tiene la tarea de educar, orientar, socializar, mediar, planificar y coordinar acciones directas para deshabilitar las prácticas laborales que ejecutan los niños y niñas frente a la precariedad laboral de las sociedades adultocéntricas que enfrentan las crisis económicas, sanitarias y sociales. Así mismo es preciso reconocer que cada una estas tareas ameritan el compromiso y participación activa tanto de los usuarios directos como de cada uno de sus familiares, dando la oportunidad para que se vuelvan gestores de su propio cambio.

En este sentido la literatura consultada refiere que "la eficacia de la intervención del Trabajador Social no sólo depende de la calidad de los recursos sociales previstos por las políticas gubernamentales, sino de lo que la persona misma es capaz de invertir como esfuerzo, motivación y deseo de salir adelante" (De Robertis, 2018).

Dentro del área de protección social, al momento de intervenir con niños, niñas y adolescentes "el trabajador social debe buscar alternativas orientadas al desarrollo de intervenciones individuales con el objetivo de revincular a los niños y niñas con su 
comunidad de origen ya sea su familia biológica o ampliada" (Silva, 2014). En este sentido el uso de una metodología apropiado es de vital importancia, en donde los modelos, técnicas y herramientas faciliten la intervención, el modelo sistémico se convierte en una guía y orientación para trabajar con la familia y mejorar su dinámica, por otra parte, el modelo de gestión de casos permite que el usuario desarrolle y ponga en práctica habilidades necesarias para mejora su bienestar.

En base a las consideraciones anteriores (Aragón, 2019) el uso del Modelo Sistémico ha sido de gran ayuda para su tratamiento el conocer las contradicciones familiares permite el cambio de sus integrantes. Este modelo ha permitido conocer la situación de niños, niñas y adolescentes y su entorno familiar, insertándolos a espacios de recreación, atención médica y educación, mediante una labor interdisciplinaria que ha generado procesos en bienestar del NNA y su colectividad. (pág. 3)

Finalmente, se hace necesario resaltar que el trabajador social interviene con este tipo de problemática conforme a la norma técnica de trabajo infantil, en donde el MIES como ente rector de la política pública en el ámbito social y económico tiene el objetivo contribuir al desarrollo integral de los grupos vulnerables.

Por tanto, las funciones que debe desempeñar el trabajador/a social son:

- Poner en conocimiento de la autoridad judicial o administrativa competente cuando hay situación de vulneración de derechos.

- Articular y elaborar informes sociales.

- Planificar, liderar y articular acciones de coordinación y articulación intersectorial para la restitución de derechos de los NNA en trabajo infantil.

- Articular acciones en el Distrito de Educación para garantizar la inserción, permanencia y promoción del NNA al sistema educativo formal o flexible.

- Participar de las reuniones, talleres, espacios de articulación y/o planificación convocadas por el MIES.

- Participar de las reuniones de análisis de caso a fin de informar y evaluar el avance.

- Mantener un expediente individual completo y actualizado de los NNA, entre otros.

\section{Metodologia.}

Se desarrolló una investigación de tipo descriptiva, con un enfoque cuali-cuantitativo, como técnicas se aplicó la encuesta a los habitantes de las Parroquias Picoazá y San Pablo del Cantón Portoviejo y la entrevista a la Trabajadora Social de la Fundación Paso a Paso, los instrumentos que facilitaron la recopilación de información fueron la guía de preguntas y el cuestionario. La población estuvo constituida por 20 habitantes de la parroquia Picoazá y 20 habitantes de la parroquia San Pablo cantón Portoviejo y la Trabajadora Social de la fundación Paso a Paso, como muestra se tomó el 100\% de la población. 


\section{Resultados.}

Resultados de la encuesta aplicada a los habitantes de las parroquias Picoazá y San Pablo

Tabla 1. ¿Cómo considera usted al trabajo infantil?

\begin{tabular}{|c|c|c|}
\hline INDICADORES & $\begin{array}{l}\text { FRECUEN } \\
\text { CIA }\end{array}$ & $\begin{array}{l}\text { PORCENT } \\
\text { AJE }\end{array}$ \\
\hline Como aporte económico favorable de su hijo a la familia & 16 & $40 \%$ \\
\hline $\begin{array}{l}\text { Una problemática que afecta al desarrollo de los niños en } \\
\text { todo ámbito }\end{array}$ & 5 & $13 \%$ \\
\hline $\begin{array}{l}\text { Como una oportunidad para mejorar los ingresos } \\
\text { económicos a su familia. }\end{array}$ & 13 & $33 \%$ \\
\hline Una vulneración de derechos de los niños & 6 & $15 \%$ \\
\hline Total & 40 & $100 \%$ \\
\hline
\end{tabular}

Elaborado por: Mendoza (2021)

Fuente: habitantes de las parroquias Picoazá y San Pablo

Se puede evidenciar que el $40 \%$ de los habitantes encuestados, indican que el trabajo infantil es un aporte económico favorable para la familia, seguido de un 33\% que lo considera como como una oportunidad para mejorar los ingresos económicos a su familia, un $15 \%$ como una vulneración de derechos de los niños y un $13 \%$ expresa que es una problemática que afecta al desarrollo de los niños en todo ámbito.

Tabla 2. ¿Considera usted que el Trabajador Social cuenta con las habilidades para intervenir en este tipo de problemática

\begin{tabular}{lll}
\hline INDICADORES & FRECUENCIA & PORCENTAJE \\
\hline Si & 28 & $70 \%$ \\
\hline No & 12 & $30 \%$ \\
\hline Total & 40 & $100 \%$
\end{tabular}

Elaborado por: Mendoza (2021)

Fuente: habitantes de las parroquias Picoazá y San Pablo

En este sentido, el 70\% de los encuestados respondieron que el profesional en Trabajo Social es la persona ideal para intervenir con esta problemática, mientras que el $30 \%$ considera que no lo es.

Tabla 3.¿Cree usted que el trabajador social ha intervenido satisfactoriamente con los niños con el tema de trabajo infantil?

\begin{tabular}{lll}
\hline INDICADORES & FRECUENCIA & PORCENTAJE \\
\hline Si & 25 & $63 \%$ \\
\hline No & 15 & $38 \%$ \\
\hline Total & 40 & $100 \%$
\end{tabular}

Elaborado por: Mendoza (2021)

Fuente: habitantes de las parroquias Picoazá y San Pablo 
Los resultados muestran que el $68 \%$ de los habitantes encuestados contestaron que el/la Trabajador/a Social ha intervenido satisfactoriamente con esta problemática y un $38 \%$ expresó que no lo ha hecho.

Tabla 4.¿Cómo considera la labor del trabajador social frente al trabajo infantil?

\begin{tabular}{lll}
\hline INDICADORES & FRECUENCIA & PORCENTAJE \\
\hline Muy buena & 20 & $50 \%$ \\
\hline Buena & 12 & $30 \%$ \\
\hline Regular & 5 & $13 \%$ \\
\hline Mala & 3 & $8 \%$ \\
\hline Total & 40 & $100 \%$
\end{tabular}

Elaborado por: Mendoza (2021)

Fuente: habitantes de las parroquias Picoazá y San Pablo

Se presenta en los resultados que el 50\% de la población encuestada respondió que la intervención del profesional en Trabajo Social con la problemática del trabajo infantil es muy buena, seguido del $30 \%$ manifestó que es buena, el 13\% sostuvo que es regular y el $8 \%$ indicó que es mala.

Tabla 5. ¿Cuáles son las actividades que hace el trabajador social para disminuir el trabajo infantil?

\begin{tabular}{lll}
\hline INDICADORES & FRECUENCIA & PORCENTAJE \\
\hline Talleres de recreación & 5 & $13 \%$ \\
\hline Capacitación a padres & 13 & $33 \%$ \\
\hline Actividades de integración con la familia del niño & 7 & $18 \%$ \\
\hline Capacitación a los niños & 15 & $38 \%$ \\
\hline Total & 40 & $100 \%$
\end{tabular}

Elaborado por: Mendoza (2021)

Fuente: habitantes de las parroquias Picoazá y San Pablo

Como se aprecia, el 38\% de los habitantes encuestados indicaron que el trabajador social realiza capacitación a los niños a fin de disminuir el trabajo infantil, el 33\% afirmó que hace capacitación a los padres, el 18\% mencionó que realiza actividades de integración con la familia y el $13 \%$ expresó que hace talleres de recreación.

\section{Resultados de la entrevista aplicada a la trabajadora social.}

Frente a la situación que se está atravesando, se realizó la entrevista mediante una videollamada, con la profesional en Trabajo Social de la fundación Paso a Paso y al preguntarle ¿Cómo considera usted el trabajo infantil? respondió "Suele definirse como el trabajo que priva a los niños, niñas y adolescentes de su niñez, muchas veces es perjudicial para el desarrollo físico y psicológico del niño, afectando totalmente su integridad. Es un trabajo prohibido para menores de edad”. 
Como siguiente pregunta ¿Cómo aporta usted en la prevención del trabajo infantil? expresó "El trabajo preventivo que se hace para que no se incremente, es el fortalecimiento de capacidades y habilidades, no sólo se trabaja con los niños, sino que también con la familia, se articula servicios como los de salud, educación entre otros. En este sentido se hace un plan de atención a los niños y su familia".

Del mismo modo con la interrogante ¿Las edades y el género que más predominan en el trabajo infantil son? manifestó "Las edades están entre 5 a 16 años y del género es equitativo, anteriormente habían solo niños; aunque no haya un informe estadístico sobre ello, se puede considerar que es equitativo actualmente".

Como siguiente pregunta ¿Desde su experiencia, Cuales son los trabajos más comunes que realizan los menores en el cantón? ella indicó "La recolección de basura, la venta de caramelos que es el comercio informal, muchos niños ayudan en los mercados con respecto a la limpieza de vegetales, en los camales, sobre todo el comercio informal, hay padres que envían a sus hijos a vender caramelos".

Seguido de ello, se le preguntó ¿Cuáles son las normativas legales que sustentan su intervención como profesionales? y contestó "Está la norma técnica sobre erradicación del trabajo infantil que está emitida por el órgano rector MIES, hay ítems que indican cuál es el rol de cada técnico de cada área porque es una triada: el trabajador social, el psicólogo y un educador o llamados también promotores".

Respecto al proceso que aplica en su intervención se le preguntó ¿Cuál es proceso metodológico que aplica usted en su intervención ante el trabajo infantil? refirió "Diagnóstico e intervención atención integral a niñas, niños y adolescentes. Intervención con la familia (acompañamiento familiar) encuentros comunitarios seguimiento y evaluación coordinación intersectorial. Desvinculación del trabajo infantil. Inserción y permanencia en el sistema educativo y actividades extracurriculares. Seguimiento post desvinculación. Verificar que no realiza ninguna actividad de trabajo infantil. Desvinculación total de la actividad de trabajo infantil. Cierre del caso. Ambiente favorable para su desarrollo psicosocial y en goce de sus derechos. Cuidado y protección de su familia".

Frente a la interrogante ¿Aplica usted un modelo de intervención ante esta problemática? manifestó "Hay un modelo de gestión donde se hace un diagnóstico de intervención integral en niños, niñas y adolescentes, se hace acompañamiento familiar con la familia del niño o niña que es reportado. La nómina de niñas la entrega el MIES y en base a ello se hace la intervención, también se hace encuentros comunitarios, hay que hacer articulaciones con los personajes claves de la comunidad o localidades en las que se intervienen, se hace seguimiento y evaluación cada 3 meses de las temáticas que se han planteado o de los objetivos con la familia y con el niño, se hace una coordinación intersectorial con los centros de salud, cuerpo de bomberos, policía, juntas parroquiales y todo personaje clave o institución que pueda aportar con este trabajo. El máximo tiempo de intervención es de 18 meses, luego de todo el proceso de intervención hay que 
hacer una desvinculación del niño del programa siempre y cuando el niño tenga las condiciones básicas como estar estudiando y que la familia sea su apoyo. Anteriormente se hacían actividades extracurriculares que se hacían actividades específicas para fortalecer algunos temas con los niños y la familia, pero ahora con la pandemia es complicado, aunque se las tiene que hacer virtualmente; luego de ello se hace el cierre de caso donde se verifica que el ambiente sea favorable para el desarrollo psicosocial del niño y esté gozando de sus derechos y del cuidado y protección de su familia”.

Respecto a sus funciones, se le preguntó ¿Cuáles son las principales funciones que usted desarrolla en su intervención?, ella contestó "Realizar expedientes del niño con sus documentos habilitantes, de acuerdo al listado que da el MIES; abordaje con datos específicos de cada niño; aplicar fichas de diagnóstico socio-económico; ejecutar el plan integral de niños, niñas y adolescentes y una propuesta de intervención con la familia; plan de seguimiento, plan de evaluación y el cierre de caso. Poner en conocimiento a las autoridades sobre algún caso de violencia o maltrato; como se mencionó anteriormente, articular servicios. Promoción de derechos de niños; talleres en la comunidad".

Frente a la pregunta ¿Las técnicas e instrumentos que aplica en su intervención son?, expresó "Como técnica, se hace la observación, diálogo participativo con los padres, visitas domiciliarias; como instrumentos se hacen informes ya sean trimestrales, semestrales o anuales, el diario de campo".

Conforme a la intervención con los padres de los niños se le preguntó ¿Cuál es la intervención que usted realiza con los padres de familia o familiares de los niños, niñas y adolescentes víctimas de trabajo infantil?, respondió "Básicamente es sensibilizar y fortaleces capacidades que abarca desde desarrollo personal (técnicas para empoderar a los padres para aclarar roles, funciones, definir la importancia del buen trato, el autoestima) hasta el desarrollo social, va de la mano con el desarrollo personal, pero trata de relaciones familiares y relaciones con sus pares, es decir, con niños de la misma edad".

Se le preguntó ¿Considera que ha mejorado la calidad de vida de los niños y padres con su intervención social?, ella indicó "Sí, realmente se puede evidenciar el mejoramiento de la calidad de vida con las que se ha trabajado, los cambios que la familia genera son notorios, no solo en las actitudes del niño, sino de la familia en general como por ejemplo el buen trato, el afecto, esto hace que los niños tengan un sentido de pertinencia".

\section{Discusión}

En relación al trabajo infantil, Gavilánez (2017), refiere que "las condiciones de pobreza de las familias de estos sectores de la ciudad de Quito, obligan a que los niños y niñas deban integrarse al ámbito laboral desde tempranas edades, aún a costa de su propia seguridad e integridad" (p. 90). 
En cuanto a los riesgos del trabajo infantil, Coro (2016) menciona que si "los padres de familia de los niños de la Plaza de San Francisco de Quito hubiesen tenido un conocimiento exhaustivo de los riesgos a los cuales se exponen sus hijos al laborar, no les obligarían a trabajar en las calles" (p.65).

Por su parte, Sinchi (2018) aporta que "es importante recalcar que existen actividades propias de la profesión, en la que el o la Trabajadora Social aporta a la prevención y erradicación progresiva del trabajo infantil, y garantizando los derechos de los niños, niñas y adolescentes" (80).

Del mismo modo, Jiménez (2018) concuerda que "todo trabajador social debe fomentar la concientización de que los niños, niñas y adolescentes deben asumir sus derechos y responsabilidades, principalmente en la inserción en el sistema educativo" (p.8).

La literatura consultada refiere que "la eficacia de la intervención del Trabajador Social no sólo depende de la calidad de los recursos sociales previstos por las políticas gubernamentales, sino de lo que la persona misma es capaz de invertir como esfuerzo, motivación y deseo de salir adelante" (De Robertis, 2018).

\section{Conclusiones.}

- El Trabajo Infantil es una problemática que afecta al potencial y la dignidad de los niños, además, es perjudicial para su desarrollo físico, psicológico y educativo. Reduce en los niños la oportunidad de estudiar y de superación; una de las causas que conllevan a que los niños trabajen a temprana edad son: la situación económica, ser miembros de familias disfuncionales y el agotamiento físico de los niños.

- El accionar del trabajador social frente a esta problemática es importante, ya que a través de sus técnicas e instrumentos que aplica, hace conocer y palpar la situación a profundidad del niño mediante la elaboración de informes, la articulación con instituciones competentes como educación, salud, entre otros; con la gestión, liderazgo y dinamismo que posee para la restitución de derechos, a ejecutar un plan de acción para que su calidad de vida mejore y los niños, niñas y adolescentes puedan gozar de sus derechos y disfrutar de su niñez.

- El trabajador social cuenta con un modelo de gestión institucional para intervenir en el campo del trabajo infantil, accionar que se fortalece con los modelos, técnicas e instrumentos propios de la profesión.

- Las actividades que desarrolla el trabajador social tanto con los niños como con su familia son talleres educativos y preventivos, jornadas lúdicas recreativas y de integración familiar.

\section{Referencias bibliográficas.}

Aragón, M. \& Oña, P. (2019). Trabajo Social en los procesos de vigilancia y seguimiento del Proyecto de Erradicación del Trabajo Infantil MIES, periodo septiembre 2016 
- febrero 2017. Tesis, Universidad Central del Ecuador. http://www.dspace.uce.edu.ec/handle/25000/18834

Aragundi, S., Menéndez, F. (2021). Intervención del trabajador social en el programa de erradicación del trabajo infantil. 2020. Revista Caribeña de Ciencias Sociales. 10(2), 44-61. https://www.eumed.net/es/revistas/caribena/febrero21/erradicacion-trabajo-infantil

Cervantes, M. S. (2017). TRABAJO SOCIAL Y PROTECCIÓN INTEGRAL. Obtenido de https://classroom.google.com/c/MjI1MDYyMDk1NzBa/a/MTkwNzIyMzc0NzN a/details http://www.dspace.uce.edu.ec/bitstream/25000/18834/1/T-UCE-0013CSH-111.pdf

Código de la niñez y adolescencia. (2014). https://www.igualdad.gob.ec/wpcontent/uploads/downloads/2017/11/codigo_ninezyadolescencia.pdf

Constitución de la República del Ecuador.

(2009). https://www.wipo.int/edocs/lexdocs/laws/es/ec/ec030es.pdf

Coro, J. (2016). El trabajo infantil y el impacto en el rendimiento escolar de los niños y niñas de la Plaza de San Francisco: Una mirada desde el Principio de corresponsabilidad del Estado y la Familia, período 2014 - 2015. Universidad Central del Ecuador. http://www.dspace.uce.edu.ec/bitstream/25000/10618/1/TUCE-0017-TS014-2016.pdf

De Robertis, C. (2018). Los Derechos Humanos, principios orientadores de la práctica del Trabajo Social . Trabajo Social Global, 8, 19-34. https://digibug.ugr.es/bitstream/handle/10481/51338/TSG\%20V8_extra_3\%20R obertis.pdf?sequence $=1 \&$ isAllowed $=y$.

INEC. (2015). Trabajo infantil en Ecuador: Hacia un entendimiento integral de la problemática, https://www.ecuadorencifras.gob.ec/documentos/webinec/Bibliotecas/Libros/Trabajo_Infantil_en_Ecuador.pdf

ILO. (2018). Poner fin al trabajo infantil para 2025: revisión de políticas y programas. https://www.ilo.org/ipec/Informationresources/WCMS_653987/lang-en/index.htm

Jaramillo, M. (2017). Veinte años de erradicación del trabajo infantil en Colombia. Infancias Imágenes, 16(1),

43-59. https://revistas.udistrital.edu.co/index.php/infancias/article/view/11464

Jiménez, K., Jhonson Ramírez, I. (2018). La intervención del trabajador social en la política pública para la erradicación del trabajo infantil del Ecuador. Tesis de pregrado, Universidad Técnica de Machala. http://repositorio.utmachala.edu.ec/handle/48000/12770 
Leyva, M., \& Pichardo, S. (2016). ¿Un mundo sin trabajo infantil? El Cotidiano, (197), 73-81. https://www.redalyc.org/pdf/325/32545857010.pdf.

Macri, M. y Uhart, C. (Coords), (2017). Niñas, niños y adolescentes trabajadores. Trayectorias socioeducativas y laborales. Buenos Aires, Argentina: Editorial Biblos.

Morán, S. (2020). El trabajo infantil en Ecuador aumentará por la pandemia, https://www.planv.com.ec/historias/sociedad/el-trabajo-infantil-ecuadoraumentara-la-pandemia

Nova, P. (2008). Trabajo infantil: los riesgos laborales en situaciones legalmente prohibidas y sus consecuencias para la salud y seguridad. Medicina y Seguridad del Trabajo, $\quad$ 54(213), 9-21. http://scielo.isciii.es/scielo.php?script=sci_arttext\&pid=S0465546X2008000400002

Ochoa, M. (2020). Intervención del trabajador social frente al trabajo infantil en familias asentadas en barrios periféricos, Tesis, Universidad Técnica de Machala. http://repositorio.utmachala.edu.ec/handle/48000/15994.

Tesis,

Universidad

Central

del

Ecuador. http://www.dspace.uce.edu.ec/handle/25000/15863

Pérez, M. (2017). La participación infantil en las actividades productivas en México. Ciudad de México, México: El Colegio de México.

Sandoval, A. (2007). Trabajo infantil e inasistencia escolar. Revista Brasileira de Educação. Recuperado de: https://www.scielo.br/scielo.php?pid=S1413$24782007000100006 \&$ script $=$ sci_arttext\&tlng=es, 12(34), 68-80.

Silva, R. (2014) Los chicos en la calle, primera edición, Editorial Buenos Aires, Argentina. http://200.12.169.19/bitstream/25000/10618/1/T-UCE-0017-TS0142016.pdf

Sinchi, D. (2018). El trabajo infantil desde un abordaje de Trabajo Social en la ciudad de Cuenca. Universidad de Cuenca. http://dspace.ucuenca.edu.ec/bitstream/123456789/29891/1/Trabajo\%20de\%20 Graduacion.pdf

Unicef. (2018). Situación del trabajo infantil en Ecuador, https://www.unicef.org/ecuador/comunicados-prensa/situaci\%C3\%B3n-deltrabajo-infantil-enecuador\#: :text=Evoluci\%C3\%B3n\%20del\%20trabajo\%20infantil\%20en,a\%20 un $\% 208 \% 2 \mathrm{C} 6 \% 25$ 


\section{PARA CITAR EL ARTÍCULO INDEXADO.}

Mendoza Loor, M. I., \& Loor Lino, L. E. (2021). Labor de trabajador social frente al trabajo infantil de los niños, niñas y adolescentes en el cantón Portoviejo. AlfaPublicaciones, 3(4), 6-19. https://doi.org/10.33262/ap.v3i4.106

\section{Ciencia}

El artículo que se publica es de exclusiva responsabilidad de los autores y no necesariamente reflejan el pensamiento de la Revista Alfa Publicaciones.

El artículo queda en propiedad de la revista y, por tanto, su publicación parcial y/o total en otro medio tiene que ser autorizado por el director de la Revista Alfa Publicaciones.
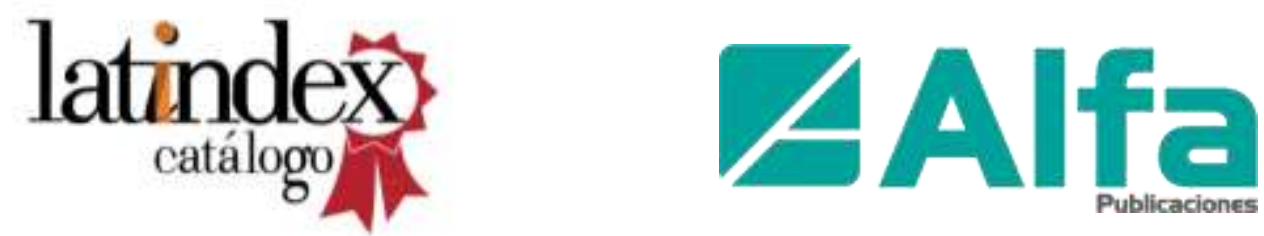